Case Report

\title{
Three-Year Successful Cinacalcet Treatment of Secondary Hyperparathyroidism in a Patient with X-Linked Dominant Hypophosphatemic Rickets: A Case Report
}

\author{
Diana Grove-Laugesen and Lars Rejnmark \\ Aarhus University Hospital, Department of Endocrinology and Internal Medicine, Tage-Hansens Gade, 8000 Aarhus C, Denmark \\ Correspondence should be addressed to Diana Grove-Laugesen; jegrov@rm.dk
}

Received 9 December 2013; Accepted 3 January 2014; Published 10 February 2014

Academic Editors: M. A. Boyanov and W. V. Moore

Copyright (C) 2014 D. Grove-Laugesen and L. Rejnmark. This is an open access article distributed under the Creative Commons Attribution License, which permits unrestricted use, distribution, and reproduction in any medium, provided the original work is properly cited.

\begin{abstract}
Hypophosphatemic rickets (HR) is a rare inherited disorder characterized by a classic rickets phenotype with low plasma phosphate levels and resistance to treatment with vitamin D. Development of secondary hyperparathyroidism (SHPT) as a direct consequence of treatment is a frequent complication and a major clinical challenge, as this may increase risk of further comorbidity. Cinacalcet, a calcimimetic agent that reduces the secretion of PTH from the parathyroid glands, has been suggested as adjuvant treatment to SHPT in patients with HR. However, only two papers have previously been published and no data are available on effects of treatment for more than six months. We now report a case of 3-year treatment with cinacalcet in a patient with HR complicated by SHPT. A 53-year-old woman with genetically confirmed X-linked dominant hypophosphatemic rickets developed SHPT after 25 years of conventional treatment with alfacalcidol and phosphate supplements. Cinacalcet was added to her treatment, causing a sustained normalization of PTH. Ionized calcium decreased, requiring reduction of cinacalcet, though asymptomatical. Level of phosphate was unchanged, but alkaline phosphatase increased in response to treatment. Cinacalcet appeared to be efficient, safe, and well tolerated. We recommend close control of plasma calcium to avoid hypocalcemia.
\end{abstract}

\section{Introduction}

Familial hypophosphatemic rickets (HR) is a rare inherited disorder characterized by renal phosphate wasting and inappropriately low levels of calcitriol causing hypophosphatemia and abnormal bone mineralization, first described by Albright [1]. The typical phenotype is characterized by severe bone deformities and growth retardation presenting in childhood with bowing of legs and shortness of length. In adulthood, the disease is often complicated by osteomalacia with bone pain and arthralgias due to joint deformities and enthesopathies. Occurrence of dental problems like tooth abscesses is also common $[2,3]$.

In most instances, HR is caused by mutations in genes affecting the metabolism of fibroblast growth factor 23 (FGF23) [4]. The prevailing form of HR is X-linked dominant hypophosphatemia (XLH) caused by an inactivating mutation of the PHEX gene [5].
Medical treatment of HR includes activated vitamin D analogues and supplements with high amounts of oral inorganic phosphate salts which heal osteomalacia, but neither corrects the biochemical abnormalities nor the bone deformities [6]. The renal phosphate leak is not improved; in contrary, treatment has been shown to increase levels of FGF23 [7]. Also, phosphate supplements tend to increase PTH levels [8] and secondary or even tertiary hyperparathyroidism may develop in response to treatment [9]. Secondary hyperparathyroidism (SHPT), reported in as many as $25 \%$ of treated patients, is associated with an increased risk of nephrocalcinosis and hypertension and may require cessation of treatment [10].

Cinacalcet is a calcimimetic agent which increases the sensitivity of the calcium-sensing receptor (CaSR) to extracellular calcium, causing reduced release of PTH $[11,12]$. In the setting of chronic kidney disease, long term treatment 
with cinacalcet has proven efficient in controlling biochemical parameters associated with SHPT [13]. A study of the acute effect of cinacalcet in XLH demonstrated reduced levels of PTH and increased renal phosphate reabsorption [14]. However, no long term data on the effects of cinacalcet on calcium-phosphate homeostasis in XLH patients with SHPT are available. Here, we report the outcome of threeyear treatment with cinacalcet in a patient with XLH and SHPT.

\section{Case Report}

A white Caucasian woman, born in 1954, developed SHPT at the age of 53 approximately 25 years after onset of treatment of XLH. As a child, the patient was in 1957 referred for bowing of lower legs, interpreted as a physiological phenomenon that would correct itself. At the age of 14 , the patient was diagnosed with osteomalacia and started treatment with cholecalciferol. At the age of 22 the patient was osteotomized in both tibiae and a diagnosis of familial hypophosphatemic rickets was suspected. The patient was referred to a medical department where she presented with shortness of stature, discrete bowing of legs, and increased lumbar lordosis. She had a history of tooth abscesses and complained of patellar subluxations and knee and hip arthralgias. X-ray demonstrated early signs of osteoarthritis in knees and hips. The diagnosis was established in 1977 based on biochemical and urine analysis showing a reduced renal tubular maximum reabsorption rate of phosphate $(\mathrm{TmP} / \mathrm{GFR} 0.48 \mathrm{mmol} / \mathrm{L}$ : reference interval $0.6-1.4 \mathrm{mmol} / \mathrm{L}$ ), with no hypercalciuria (urinary calcium $2.9 \mathrm{mmol} / \mathrm{d}$ : reference interval $1.2-3.8 \mathrm{mmol} / \mathrm{d}$ ) but low plasma levels of calcitriol (16 pmol/L: reference interval 24$158 \mathrm{pmol} / \mathrm{L}$ ) in the presence of hypophosphatemia (plasma phosphate $0.6 \mathrm{mmol} / \mathrm{L}$ : reference interval: $0.8-1.5 \mathrm{mmol} / \mathrm{L})$. An iliac crest bone biopsy revealed severe osteomalacia with low bone turnover. Due to low adherence to clinical followup visits, medical treatment with phosphate supplements and cholecalciferol was not initiated until five years later when the patient was 27 years old. Cholecalciferol was exchanged for an activated vitamin D analog (alfacalcidol) two years later. Repeated bone biopsy after two years of treatment revealed healing of mineralization defect, but complaints of joint ache endured and increased, necessitating knee and hip replacement in 2010 and 2012, respectively. At the age of 51 the patient was entitled incapacity benefit. Genetic analysis identified a missense-mutation in the PHEX-gene showing the patient to be heterozygous for c.1601 C>T, exon 15. The patient's sister and two children, a boy and a girl, share the same mutation.

In 2007 the patient was diagnosed with SHPT which was not corrected by adjustment of therapy. Being severely symptomatic, continued treatment was required, and in March 2010 the patient consented to experimental treatment with cinacalcet in an attempt for curing her SHPT. Thirty mg of cinacalcet was added to her treatment as displayed in Figure 1. An attempt at increasing dosage to $60 \mathrm{mg}$ was abandoned because of a fall in plasma calcium (October 2010, Figure 1(a)). Hereafter, PTH was stable and within the reference interval until the dosage of cinacalcet was reduced
TABLE 1: Effect of cinacalcet treatment on biochemical indices of calcium homeostasis in a patient with X-linked dominant hypophosphatemia.

\begin{tabular}{|c|c|c|c|}
\hline Plasma (reference interval) & $\begin{array}{c}\text { Before } \\
\text { cinacalcet } \\
\text { treatment }\end{array}$ & $\begin{array}{l}\text { During } \\
\text { cinacalcet } \\
\text { treatment }\end{array}$ & $P$ value \\
\hline PTH, pmol/L (1.6-6.9) & $9.3 \pm 3.3$ & $5.4 \pm 3.2$ & 0.03 \\
\hline $\begin{array}{l}\text { Calcium-ionized, } \mathrm{mmol} / \mathrm{L} \\
(1.18-1.32)\end{array}$ & $1.25 \pm 0.03$ & $1.15 \pm 0.04$ & $<0.01$ \\
\hline $\begin{array}{l}\text { Phosphate, } \mathrm{mmol} / \mathrm{L} \\
(0.76-1.41)\end{array}$ & $0.84 \pm 0.10$ & $0.80 \pm 0.08$ & 0.46 \\
\hline $\begin{array}{l}\text { Alkaline phosphatase U/L } \\
(35-105)\end{array}$ & $94 \pm 7$ & $118 \pm 8$ & $<0.001$ \\
\hline Calcidiol, nmol/L (50-160) & $72 \pm 20$ & $72 \pm 8$ & 0.96 \\
\hline Creatinine, $\mu \mathrm{mol} / \mathrm{L}(45-90)$ & $58 \pm 2$ & $61 \pm 4$ & 0.17 \\
\hline
\end{tabular}

Mean plasma levels ( \pm standard deviation) in the three years prior to the start of treatment and during three years of treatment with cinacalcet. $P$ values denote difference in mean values.

prior to planned hip surgery (30 mg every second day, August 2012) resulting in elevated PTH levels that endured until dosage of cinacalcet was adjusted at $30 \mathrm{mg}$ per day (May 2013). Plasma phosphate was on average unchanged after addition of cinacalcet (Figure 1(b)). Plasma calcium was lower than prior to cinacalcet treatment but the patient did not experience hypocalcemic symptoms, and treatment was well tolerated without complaints of adverse effects (see Table 1). Following start of treatment with cinacalcet, plasma levels of alkaline phosphatase (ALP) rose significantly but stabilized (Figure 2).

\section{Discussion}

3.1. Effect on PTH and Calcium. To the best of our knowledge, this is the first report on long term use of cinacalcet as a therapeutic approach to SHPT in a patient with XLH. During the three years of treatment, PTH levels showed a sustained dose-dependent response to the treatment with cinacalcet and were maintained within the reference interval when dosage of cinacalcet was $30 \mathrm{mg}$ per day or more. This is in accordance with studies on the effect of cinacalcet in patients with primary hyperparathyroidism [15], and observations in chronic kidney disease where cinacalcet has proven efficient in the long term control of SHPT [13].

Previously, only one case report has described the use of cinacalcet in a patient with XLH complicated by SHPT, as Yavropoulou et al. [16] reported successful treatment with cinacalcet $60 \mathrm{mg}$ a day for a period of six months with sustained normalization of PTH. Total calcium levels were normal at all times with a tendency to decline, although ionized calcium levels surprisingly tended to rise. In contrast, our data suggest that cinacalcet lowers plasma calcium levels in patients with $\mathrm{HR}$, similar to the effect reported in patients with primary hyperparathyroidism [15].

We found only one clinical study on the effect of cinacalcet in patients with XLH. Alon et al. [14] studied eight children with XLH but with normal plasma levels of ionized 


\begin{tabular}{|c|c|c|c|c|c|c|}
\hline Alfacalcidol $\mu / \mathrm{d}$ & \multicolumn{6}{|c|}{2} \\
\hline Phosphate g/d & 15 & 9 & & & & 20 \\
\hline $\mathrm{CaD} \mathrm{mg}+\mu \mathrm{g} / \mathrm{d}$ & \multicolumn{6}{|c|}{$250+30$} \\
\hline Cinacalcet $\mathrm{mg} / \mathrm{d}$ & & & 60 & 30 & 15 & 30 \\
\hline
\end{tabular}

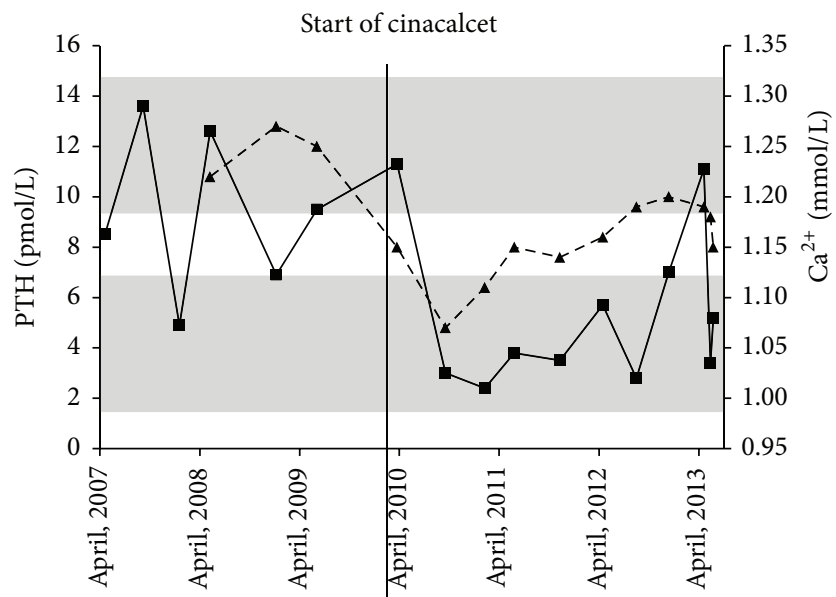

(a)

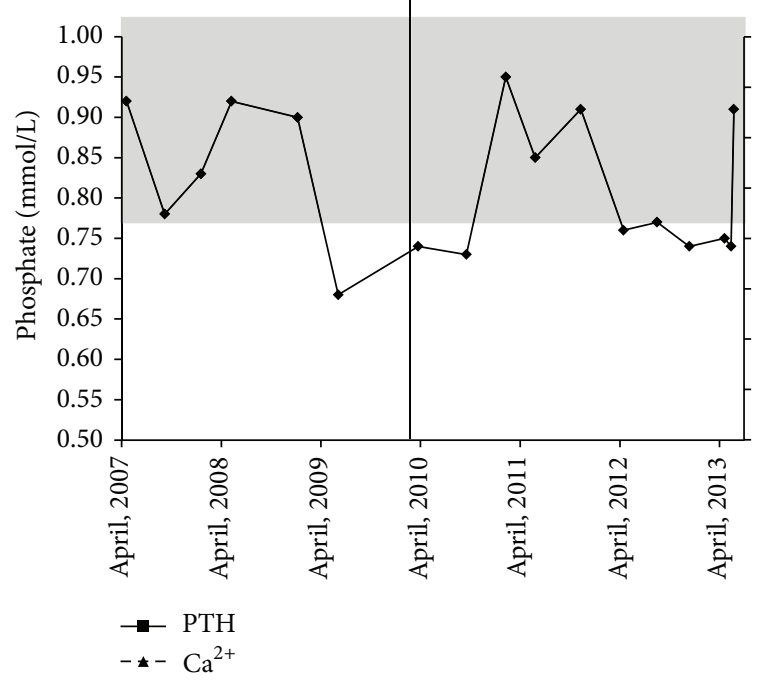

(b)

FIGURE 1: Plasma levels of PTH, ionized-calcium (Figure 1(a)), and phosphate (Figure 1(b)) three years before initiation of cinacalcet treatment and during the three years of cinacalcet treatment. Start of cinacalcet treatment, as indicated by the vertical line, was March 2010 . Top bars show dosage of medical treatment. CaD; calcium carbonate $250 \mathrm{mg}$ with $30 \mu \mathrm{g} / \mathrm{d}$ (1200 IU) of cholecalciferol. Reference intervals of plasma PTH (1.6-6.9 pmol/L) are indicated by the lower grey area and plasma $\mathrm{Ca}^{2+}(1.18-1.32 \mathrm{mmol} / \mathrm{L})$ by the upper grey area. Measurements of ionized calcium $\left(\mathrm{Ca}^{2+}\right)$ were not available prior to May 2008 (Figure 1(a)). The reference interval of plasma phosphate was $0.76-1.41 \mathrm{mmol} / \mathrm{L}$, as indicated by the grey area (Figure 1(b)).

calcium and PTH. In a run-in period of 7 days prior to the study, regular treatment with phosphate and calcitriol was discontinued. During the subsequent 48 -hour study period the patients received phosphate supplement. Alon et al. [14] demonstrated that a single dose of cinacalcet $(30$ or $60 \mathrm{mg}$, depending on weight) on day two reduced PTH and plasma calcium significantly compared to baseline values.

We measured equally low levels of calcium on only one occasion, when our patient was treated with a daily dose of $60 \mathrm{mg}$ cinacalcet (October 2010 as shown in Figure 1). It seems reasonable to assume that treatment with activated vitamin $\mathrm{D}$ analogues as in our case but which is absent in the study by Alon et al. [14] will tend to attenuate this calcium lowering effect of cinacalcet.

3.2. Effect on Plasma Phosphate. Following the reduction of plasma PTH and hence reduced renal phosphate loss, an increase in plasma phosphate may be expected. 


\begin{tabular}{|c|c|c|c|c|c|c|}
\hline Alfacalcidol $\mu / \mathrm{d}$ & \multicolumn{6}{|c|}{2} \\
\hline Phosphate g/d & 15 & 9 & 15 & 20 \\
\hline CaD mg + $\mu \mathrm{g} / \mathrm{d}$ & \multicolumn{6}{|c|}{$250+30$} \\
\hline Cinacalcet mg/d & & 30 & 60 & 30 & 15 & 30 \\
\cline { 2 - 6 } & &
\end{tabular}

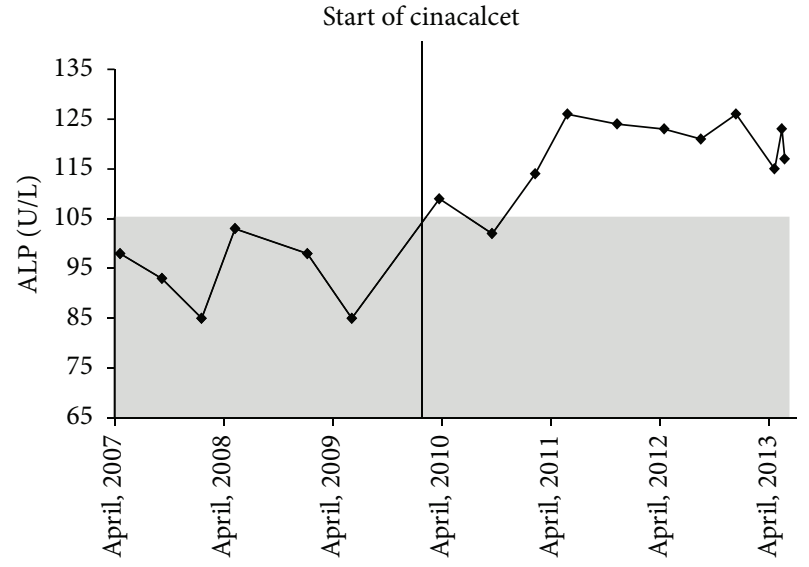

FIGURE 2: Plasma levels of alkaline phosphatase three years before initiation of cinacalcet treatment and during the three years of cinacalcet treatment. Start of cinacalcet treatment, as indicated by the vertical line, was March 2010. Top bars show dosage of medical treatment. CaD; calcium carbonate $250 \mathrm{mg}$ with $30 \mu \mathrm{g} / \mathrm{d}$ (1200 IU) of cholecalciferol. The reference interval of plasma alkaline phosphatase was 35-105 U/L as indicated by the grey area.

In a study including two patients with FGF-23 mediated tumor induced osteomalacia, adjuvant therapy with cinacalcet led to increased plasma phosphate levels and allowed a substantial reduction of calcitriol and phosphate supplements [17]. A similar phosphate-saving effect of cinacalcet has been suggested in patients with XLH [14].

However, plasma phosphate remained stable in our patient despite reduced plasma PTH levels and unchanged phosphate supplements. In the case series by Yavropoulou et al. [16], plasma phosphate as well as TmP/GFR increased. Interestingly, this occurred with a concomitant normalization of FGF-23, which most likely contributed to the observed increase in plasma phosphate levels. However, in the two patients with FGF-23 mediated tumor induced osteomalacia studied by Geller et al. [17], the increase in plasma phosphate and TmP/GFR occurred despite increased FGF-23 levels during treatment with cinacalcet. Unfortunately, we were not able to measure FGF-23 before or during treatment with cinacalcet. Further studies are needed to assess possible effects of cinacalcet on plasma phosphate and FGF-23, including whether an effect may vary according to the etiology of hypophosphatemia and between different genetic mutations in patients with HR.

3.3. Effect on ALP. Despite decreased PTH levels, we observed increased levels of ALP during cinacalcet treatment. We speculate that this may be a consequence of treatment with cinacalcet. Our patient had a stable calcidiol level and she did not suffer any fractures. Her orthopedic operation was performed 2.5 years after initiation of cinacalcet treatment. The levels of ALP were not reported in any of the previously mentioned studies of XLH patients. Reports on changes in levels of ALP during treatment with calcimimetic agents are conflicting. Several studies have established that treating SHPT in hemodialysis patients with calcimimetic agents results in a decrease in ALP [18]. However, in an open labeled study of mild PHPT approached with cinacalcet during a period of 5 years, increased ALP levels were reported [15]. Dvorak et al. [19] have demonstrated that both high levels of calcium and the CaSR agonist gadolinium stimulate signaling associated with proliferation in osteoblasts and upregulate mRNA responsible for osteoblast differentiation and mineralization. Thus, a rise in ALP could be a marker of improved bone formation induced by cinacalcet. However, bone mineral density has not been shown to increase in response to treatment with cinacalcet in patients with PHPT, suggesting that a potential bone anabolic effect is of minor importance [15]. Our findings need to be confirmed by others, and the pathophysiological mechanism should be further elucidated.

\section{Conclusion}

Long term treatment with cinacalcet for a period of three years showed sustained dose-dependent response on PTH levels, and cinacalcet appeared to be safe and well tolerated. Cinacalcet may therefore be a beneficial therapeutic option in XLH with SHPT. However, we recommend close control of plasma calcium to detect hypocalcemia which seems to be a potential adverse effect of treatment. A possible effect on plasma phosphate and alkaline phosphatase levels needs further investigations. Randomized clinical studies should be performed before cinacalcet can be recommended as standard treatment in XLH complicated by SHPT. 


\section{Conflict of Interests}

The authors declare that there is no conflict of interests regarding the publication of this paper.

\section{Authors' Contribution}

Data collection, data analysis, data interpretation, and drafting of the paper were done by Diana Grove-Laugesen and Lars Rejnmark. Diana Grove-Laugesen and Lars Rejnmark take equal responsibility for the integrity of the data analysis.

\section{Acknowledgment}

This work was supported by a grant from the Health Research Fund of Central Denmark Region.

\section{References}

[1] F. Albright, A. M. Butler, and E. Bloomberg, "Rickets resistant to vitamin D therapy," American Journal of Diseases of Children, vol. 54, pp. 529-547, 1937.

[2] G. I. Baroncelli, M. Angiolini, E. Ninni, V. Galli, R. Saggese, and M. R. Giuca, "Prevalence and pathogenesis of dental and periodontal lesions in children with X-linked hypophosphatemic rickets," European Journal of Paediatric Dentistry, vol. 7, no. 2, pp. 61-66, 2006.

[3] I. R. Reid, D. C. Hardy, W. A. Murphy, S. L. Teitelbaum, M. A. Bergfeld, and M. P. Whyte, "X-linked hypophosphatemia: a clinical, biochemical, and histopathologic assessment of morbidity in adults," Medicine, vol. 68, no. 6, pp. 336-352, 1989.

[4] J. Q. Feng, E. L. Clinkenbeard, B. Yuan, K. E. White, and M. K. Drezner, "Osteocyte regulation of phosphate homeostasis and bone mineralization underlies the pathophysiology of the heritable disorders of rickets and osteomalacia," Bone, vol. 54, no. 2, pp. 213-221, 2013.

[5] F. Francis, S. Hennig, B. Korn et al., "A gene (PEX) with homologies to endopeptidases is mutated in patients with $\mathrm{X}$ linked hypophosphatemic rickets," Nature Genetics, vol. 11, no. 2, pp. 130-136, 1995.

[6] G. I. Baroncelli, S. Bertelloni, F. Sodini et al., "Genetic advances, biochemical and clinical features and critical approach to treatment of patients with X-linked hypophosphatemic rickets," Pediatric Endocrinology Reviews, vol. 1, no. 4, pp. 361-379, 2004.

[7] E. A. Imel, L. A. DiMeglio, S. L. Hui, T. O. Carpenter, and M. J. Econs, "Treatment of X-linked hypophosphatemia with calcitriol and phosphate increases circulating fibroblast growth factor 23 concentrations," Journal of Clinical Endocrinology and Metabolism, vol. 95, no. 4, pp. 1846-1850, 2010.

[8] H. P. Krohn, G. Offermann, M. Brandis, J. Brodehl, K. Hanke, and G. Offner, "Occurrence of hyperparathyroidism in children with X-linked hypophosphatemia under treatment with vitamin D and phsophate," Advances in Experimental Medicine and Biology, vol. 81, pp. 345-351, 1977.

[9] H. Ræder, N. Shaw, C. Netelenbos, and R. Bjerknes, "A case of Xlinked hypophosphatemic rickets: complications and the therapeutic use of cinacalcet," European Journal of Endocrinology, vol. 159, no. 1, pp. S101-S105, 2008.

[10] U. S. Alon, R. Monzavi, M. Lilien, M. Rasoulpour, M. E. Geffner, and O. Yadin, "Hypertension in hypophosphatemic ricketsrole of secondary hyperparathyroidism," Pediatric Nephrology, vol. 18, no. 2, pp. 155-158, 2003.
[11] L. G. Hammerland, J. E. Garrett, B. C. P. Hung, C. Levinthal, and E. F. Nemeth, "Allosteric activation of the Ca2+ receptor expressed in Xenopus laevis oocytes by NPS 467 or NPS 568," Molecular Pharmacology, vol. 53, no. 6, pp. 1083-1088, 1998.

[12] E. F. Nemeth, M. E. Steffey, L. G. Hammerland et al., "Calcimimetics with potent and selective activity on the parathyroid calcium receptor," Proceedings of the National Academy of Sciences of the United States of America, vol. 95, no. 7, pp. 40404045, 1998.

[13] G. A. Block, K. J. Martin, A. L. M. de Francisco et al., "Cinacalcet for secondary hyperparathyroidism in patients receiving hemodialysis," The New England Journal of Medicine, vol. 350, no. 15, pp. 1516-1525, 2004.

[14] U. S. Alon, R. Levy-Olomucki, W. V. Moore, J. Stubbs, S. Liu, and L. D. Quarles, "Calcimimetics as an adjuvant treatment for familial hypophosphatemic rickets," Clinical Journal of the American Society of Nephrology, vol. 3, no. 3, pp. 658-664, 2008.

[15] M. Peacock, M. A. Bolognese, M. Borofsky et al., "Cinacalcet treatment of primary hyperparathyroidism: biochemical and bone densitometric outcomes in a five-year study," Journal of Clinical Endocrinology and Metabolism, vol. 94, no. 12, pp. 4860-4867, 2009.

[16] M. P. Yavropoulou, K. Kotsa, A. G. Psarrakou et al., "Cinacalcet in hyperparathyroidism secondary to X-linked hypophosphatemic rickets: case report and brief literature review," Hormones, vol. 9, no. 3, pp. 274-278, 2010.

[17] J. L. Geller, A. Khosravi, M. H. Kelly, M. Riminucci, J. S. Adams, and M. T. Collins, "Clinical vignette: cinacalcet in the management of tumor-induced osteomalacia," Journal of Bone and Mineral Research, vol. 22, no. 6, pp. 931-937, 2007.

[18] V. Belozeroff, W. G. Goodman, L. Ren, and K. Kalantar-Zadeh, "Cinacalcet lowers serum alkaline phosphatase in maintenance hemodialysis patients," Clinical Journal of the American Society of Nephrology, vol. 4, no. 3, pp. 673-679, 2009.

[19] M. M. Dvorak, A. Siddiqua, D. T. Ward et al., "Physiological changes in extracellular calcium concentration directly control osteoblast function in the absence of calciotropic hormones," Proceedings of the National Academy of Sciences of the United States of America, vol. 101, no. 14, pp. 5140-5145, 2004. 


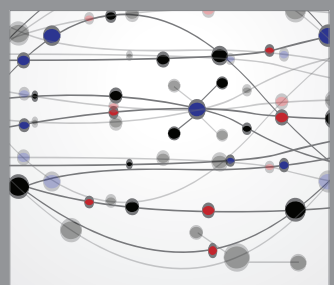

The Scientific World Journal
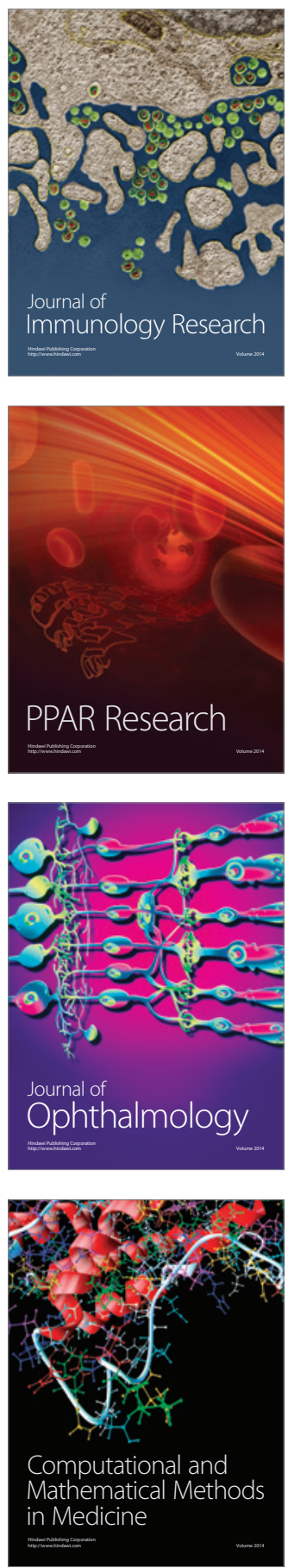

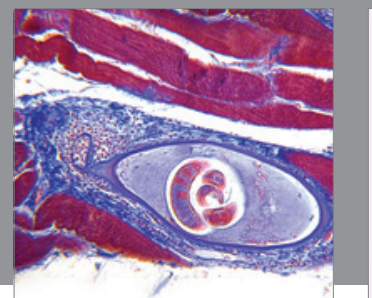

Gastroenterology

Research and Practice
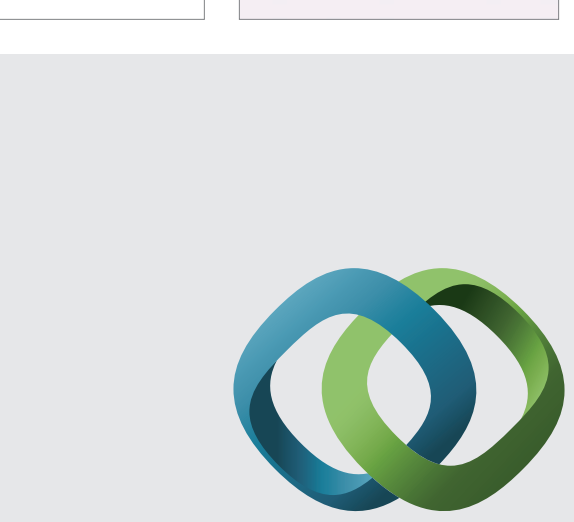

\section{Hindawi}

Submit your manuscripts at

http://www.hindawi.com
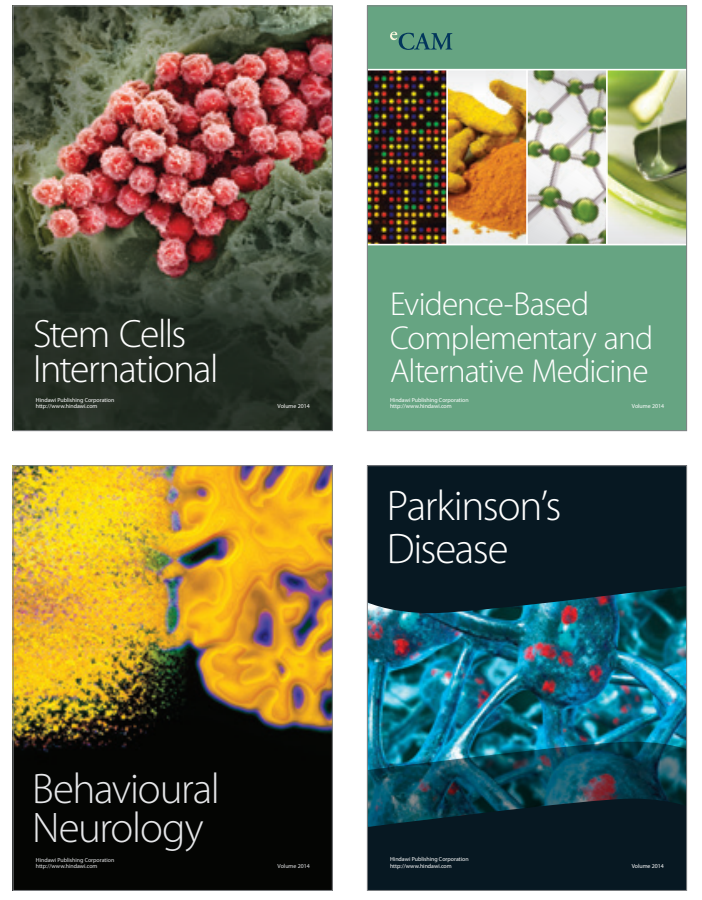
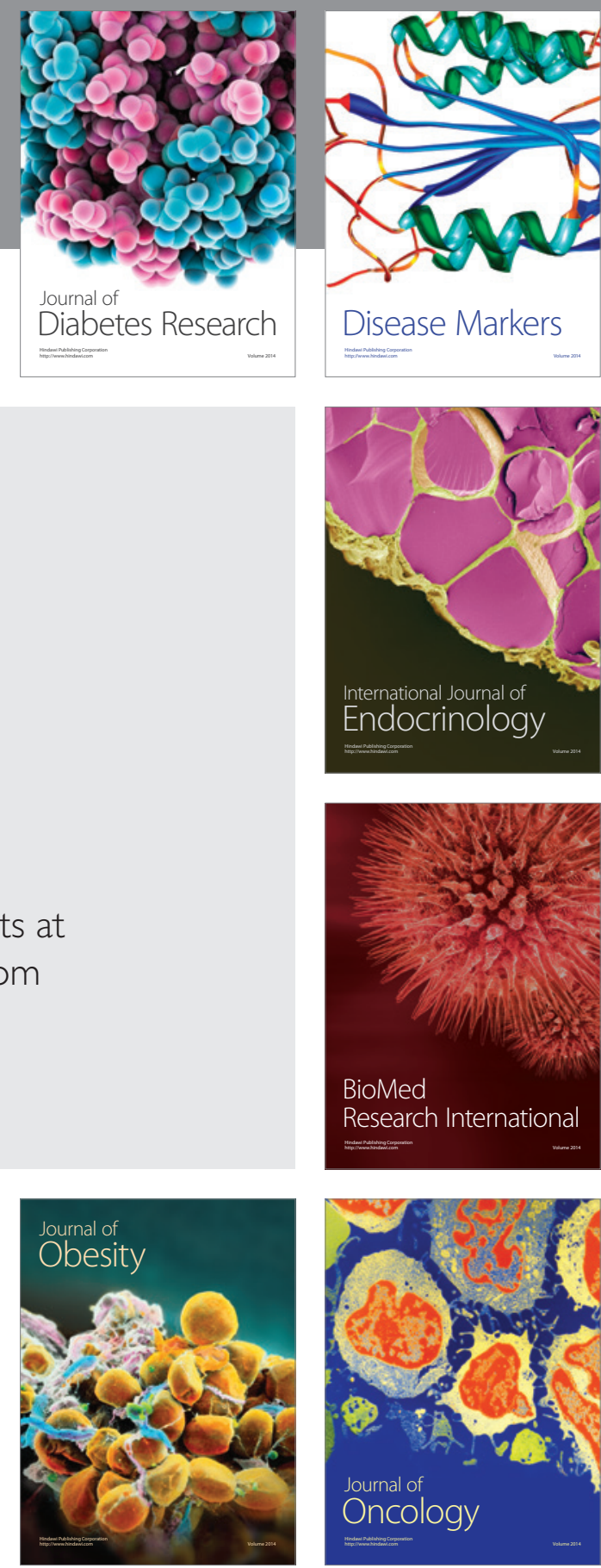

Disease Markers
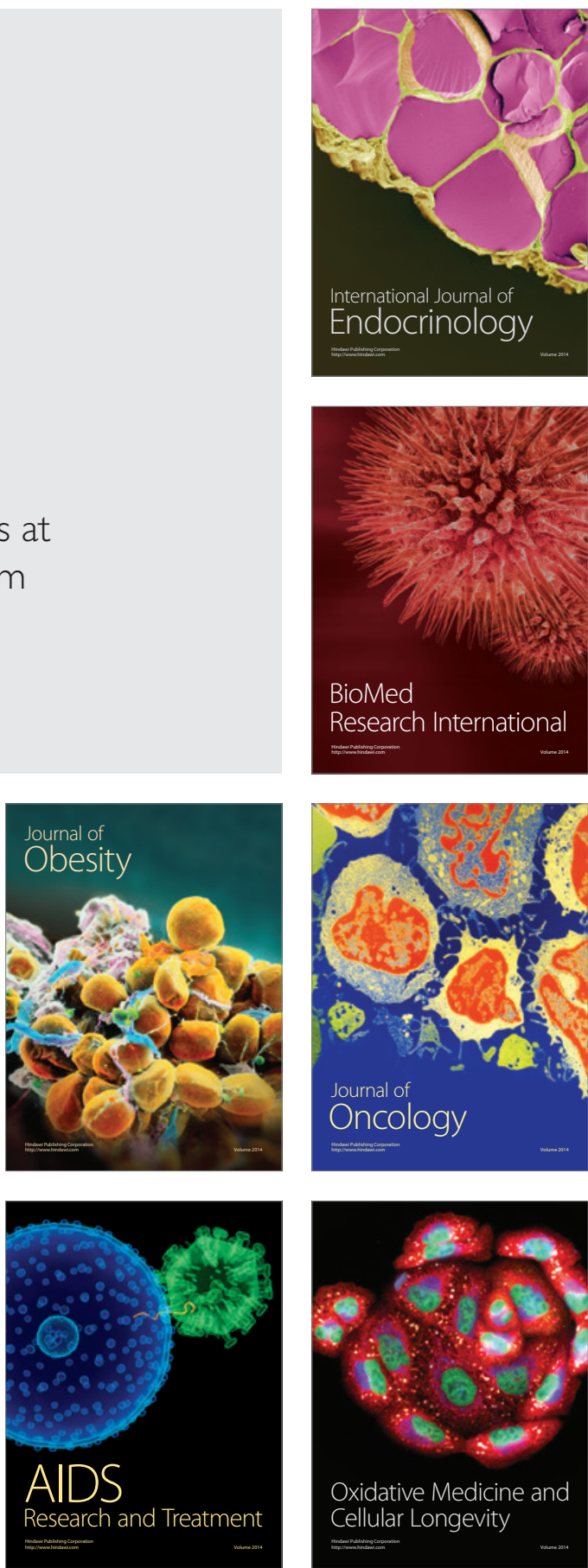